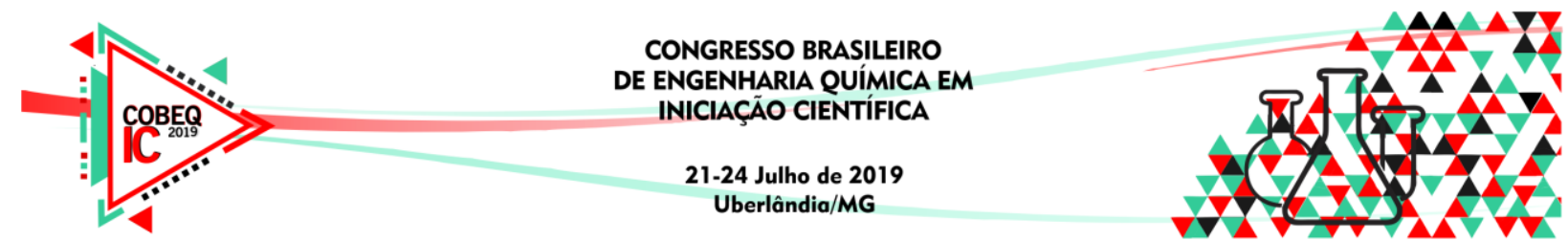

\title{
ANÁLISE E SIMULAÇÃO COMPUTACIONAL DA BIOMEMBRANA POROSA EM BIOSSENSORES PARA DETECÇÃO DE COMPOSTOS FENÓLICOS PRESENTES EM EFLUENTES URBANOS
}

\author{
Gabriellen Kelly Amaral de Oliveira $^{1}$, Simone de Aviz Cardoso ${ }^{1}$ \\ ${ }^{1}$ Universidade Federal do Pará, Instituto de Ciências Biológicas, Faculdade de Biotecnologia \\ E-mail para contato: gabriellenkelly.amaral@gmail.com
}

\begin{abstract}
RESUMO - Os biossensores são dispositivos biológicos ligados à um sistema de detecção, transdução e amplificação do sinal gerado por reação. O presente trabalho teve como objetivo a modelagem matemática de um biossensor amperométrico acoplado a um biofilme poroso plano enzimático à base de amido de mandioca para detectar fenóis presentes em efluentes urbanos. O biossensor foi dividido em duas membranas: a primeira sem enzimas imobilizadas, a segunda composta de enzima tirosinase imobilizada nos poros do biofilme que tem a finalidade de catalisar a reação. Os modelos matemáticos foram desenvolvidos através do balanço de espécies com auxílio da lei de Fick, lei de Langmuir e a lei de Faraday. As Equações resultantes foram utilizadas no COMSOL MULTIPHISICS, um software de simulação de problemas baseados em física. Assim, foram obtidos modelos matemáticos plausíveis com as necessidades de tratamento de efluentes urbanos e com a simulação foram obtidos resultados satisfatórios.
\end{abstract}

Palavras-chave: biossensor, enzimas, efluentes urbanos, porosidade.

\section{Introdução}

Biossensores são pequenos dispositivos biológicos que detectam elementos traços e pequenas quantidades de compostos (WANG, 2008, p.2048). A porosidade de biossensores está ligada à movimentação do substrato entre suas membranas. Para calcular a adsorção, utiliza-se a isoterma de Langmuir, que sugere três hipóteses a respeito da adsorção: Superfície homogênea, adsorção em monocamada e as moléculas adsorvidas não interagem entre si.

Os fenóis são compostos orgânicos nos quais o grupo $\mathrm{OH}$ estão ligados diretamente a um anel benzênico, quando exposto a temperatura ambiente é incolor e líquido, porém, geralmente é encontrado na forma sólida (OLIVEIRA, 2015, p. 2), além de serem tóxicos, podendo causar corrosão na pele.

Assim, este trabalho teve como objetivo simular um biossensor para detecção de compostos fenólicos com o objetivo de investigar a influência da porosidade das membranas que compõem o dispositivo, empregando o software COMSOL.

\section{Metodologia}

Foram desenvolvidos modelos matemáticos estruturados utilizando a equação da conservação de espécies, a lei de Fick, lei de Langmuir e lei de Faraday, que serão solucionadas computacionalmente através de softwares adequados como o software COMSOL MULTIPHISICS.

\subsection{Modelagem Matemática}




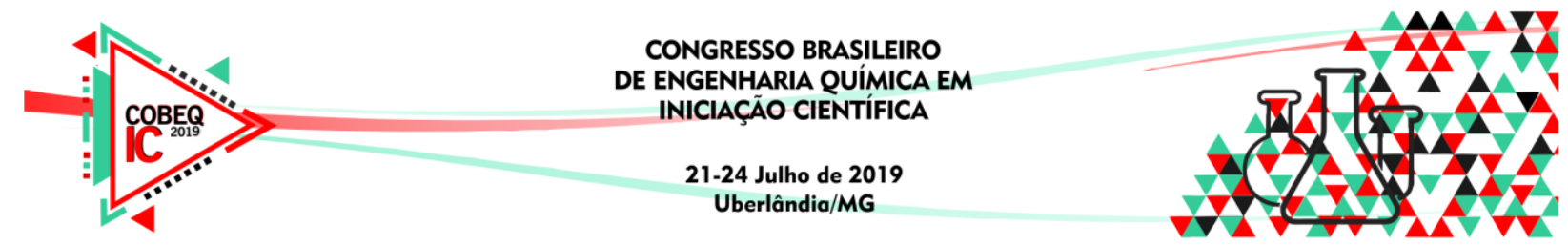

Para o biossensor, utilizou-se a tirosinase. A substância fenólica presente no substrato (S) migra por difusão na membrana porosa 1 , até encontrar a superfície da membrana porosa 2, a qual apresenta enzimas imobilizadas em sua estrutura porosa $(\mathrm{E})$. O fenol reage com a enzima imobilizada nos poros da membrana 2 conforme a reação dada pela Eq. (1).

$S+E \rightarrow P_{1}$

O produto $\left(\mathrm{P}_{1}\right)$ formado na membrana porosa 2 , migra por difusão em meio poroso até a superfície do eletrodo de trabalho, formado por nano partículas de cobre, onde reage através de oxirredução para formar o produto $\left(\mathrm{P}_{2}\right)$ e elétrons livres ( $\left.\overline{\mathrm{e}}\right)$, reação é dada pela Eq. (2).

$P_{1}+C u^{0} \rightarrow P_{2}+\bar{e}$

Os elétrons produzidos irão migrar devido ao gradiente de elétrons através de um circuito eletrônico simples, até a extremidade onde se encontra um medidor de corrente elétrica, amperímetro. As equações matemáticas obtidas através de um balanço de espécies no biossensor são obtidas em três momentos. Balanço de espécie na membrana porosa 1, balança de espécie na membrana porosa 2 e balanço de carga no eletrodo.

- Balanço de espécie na membrana porosa 1: Na membrana $1\left(0 \leq \mathrm{x} \leq \delta_{1}\right)$, a espécie $\mathrm{S}$ atravessa a membrana por difusão transiente $(\mathrm{t} \geq 0)$ não reativa, ou seja, difusão simples, conforme a Lei de Fick da difusão, dada pelas Eqs. (3):

- Balanço para a solução de efluente na membrana porosa 1:

$P_{1} \frac{\partial C_{s 1}}{\partial t}-\nabla\left(D_{s m 1} C_{s 1}\right)=0 \quad t>0 \quad 0<x<\delta_{1} ; C_{s 1}(x, t=0)=S_{0} t=0 \quad 0<x<\delta_{1}$

$\frac{\partial C_{s 1}}{\partial x}=0 \quad t>0 \quad x=0 ; \quad \frac{\partial C_{s 1}}{\partial x}=0 \quad t>0 \quad x=\delta_{1}$

$P_{1}=\varepsilon_{1}+\rho k_{p 1}$

onde, $\mathrm{C}_{\mathrm{s} 1}(\mathrm{x}, \mathrm{t})$ é o perfil de concentração de efluente ao longo da espessura da membrana porosa $1, \mathrm{~mol} . \mathrm{m}^{-3} ; \mathrm{S}_{0}$ é a concentração inicial de efluente e medida por mol.m $\mathrm{m}^{-3} ; \delta_{1}$ é a espessura da membrana 1 , em m; $\mathrm{D}_{\mathrm{sm} 1}$ é o coeficiente de difusão do efluente na membrana 1 , em $\mathrm{m}^{2} . \mathrm{s} ; \mathrm{P}_{1}$ é o produto formado na membrana porosa 1 para reagir na membrana $2 ; \mathrm{k}_{\mathrm{p} 1}$ é a razão de crescimento do poro na membrana porosa 1 , em $\mathrm{kg} \cdot \mathrm{m}^{-3} ; \varepsilon_{1}$ é a porosidade na membrana porosa 1. A equação de Langmuir pode ser definida como:

$q_{s 1}(x, t)=\frac{C_{s 1, \max }^{*} K_{L, s 1}}{1+C_{s 1}^{*}(x, t) K_{L, s 1}}$

onde, $\mathrm{q}_{\mathrm{s} 1}(\mathrm{x}, \mathrm{t})$ é a concentração de substrato adsorvido por unidade de massa de adsorvente, em $\mathrm{g} / \mathrm{g} ; \mathrm{C}_{\mathrm{s} 1}{ }^{*}(\mathrm{x}, \mathrm{t})$ é a concentração no equilíbrio do substrato em solução, em mol.m ${ }^{-3} ; \mathrm{C}^{*}{ }_{\mathrm{s} 1 \text {,max }}$ é o parâmetro que representa a concentração máxima de adsorção na monocamada, em mol.m ${ }^{3}$; $K_{L, s l}$ é o parâmetro relacionado com a energia de adsorção e dependente da temperatura, medido em $1 . \mathrm{g}^{-1}$.

- Balanço de espécie na membrana 2: Na membrana porosa $2\left(\delta_{1} \leq \mathrm{x} \leq \delta_{2}\right)$, a espécie $\mathrm{S}$ atravessa a membrana por difusão transiente $(\mathrm{t} \geq 0)$ em meio poroso com reação enzimática, conforme a Lei de Fick da difusão, dada pelas Eqs. (5): 


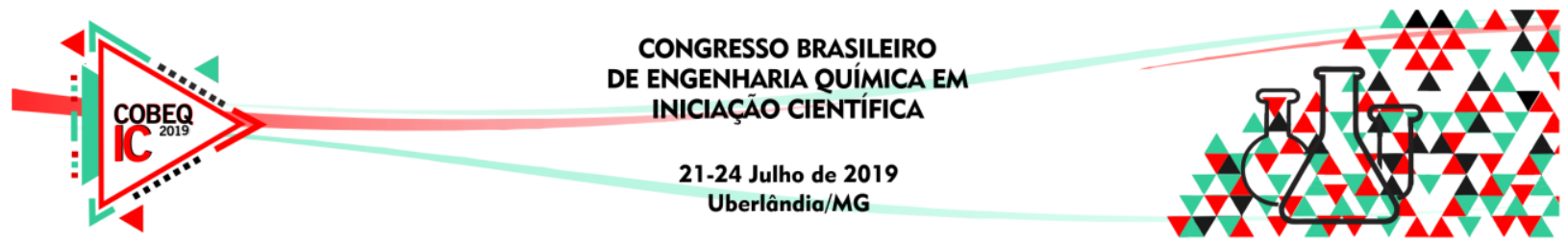

- $\quad$ Balanço para a solução de efluente na membrana porosa 2:

$P_{2} \frac{\partial C_{s 2}}{\partial t}-\nabla\left(D_{s m 2} C_{s 2}\right)=-R_{s} \quad t>0 \quad \delta_{1}<x<\delta_{2} ; C_{s 2}(x, t=0)=0 \quad t=0 \quad \delta_{1}<x<\delta_{2}$

$\frac{\partial C_{s 2}}{\partial x}=0 \quad t>0 \quad x=\delta_{1} ; \frac{\partial C_{s 2}}{\partial x}=0 \quad t>0 \quad x=\delta_{2}$

$R_{s}=\mu_{\max } \frac{C_{s 2}}{K_{m}+C_{s 2}} ; \quad q_{s 2}(x, t)=\frac{C_{s 2, \max }^{*} K_{L, s 2}}{1+C_{s 2}^{*}(x, t) K_{L, s 2}} t>0 \quad \delta_{1}<x<\delta_{2}$

$P_{2}=\varepsilon_{2}+\rho k_{p 2}$

onde, $\mathrm{C}_{\mathrm{s} 2}(\mathrm{x}, \mathrm{t})$ é o perfil de concentração de efluente ao longo da espessura da membrana porosa 2 , em mol.m ${ }^{-3} ; D_{\text {sm2 }}$ é o coeficiente de difusão de efluente na membrana porosa 2 , em $\mathrm{m}^{2} \cdot \mathrm{s}^{-1} ; \mathrm{P}_{2}$ é o produto formado na membrana porosa 2 , em mol. $\mathrm{m}^{-3} ; \mathrm{k}_{\mathrm{p} 2}$ é a razão de crescimento do poro na membrana porosa 1 , em $\mathrm{kg} \cdot \mathrm{m}^{-3} ; \varepsilon_{2}$ é a porosidade na membrana porosa 2; $\mathrm{q}_{\mathrm{s} 2}(\mathrm{x}, \mathrm{t})$ é a concentração de substrato adsorvido por unidade de massa de adsorvente, em $\mathrm{g} / \mathrm{g} ; \mathrm{C}_{\mathrm{s} 2}{ }^{*}(\mathrm{x}, \mathrm{t})$ é a concentração no equilíbrio do substrato em solução, medido em mol.m ${ }^{-3} ; \mathrm{C}_{\mathrm{s} 2 \text {,max }}^{*}$ é o parâmetro que representa a concentração máxima de adsorção na monocamada, em mol.m ${ }^{-3} ; K_{L, s 2}$ é o parâmetro relacionado com a energia de adsorção e dependente da temperatura, em $1 . \mathrm{g}^{-1}$; $\mathrm{R}_{\mathrm{S}}$ é a lei de velocidade de reação conforme o modelo de reação enzimática de Michaeles-Menten, e é dada pela Eq. (5 e), em mol. $\mathrm{m}^{-3} \cdot \mathrm{s}^{-1}$, na qual $\mu_{\max }$ é a constante de velocidade máxima dada por $\mathrm{s}^{-1}, \mathrm{~K}_{\mathrm{m}}$ é constante de Michaeles dada por mol.m ${ }^{-3}$, e $\mu_{\max }$ é representado pela Eq. (6):

$\mu_{\max }=K_{3} \times E_{t}$

$\mathrm{K}_{3}$ é a constante cinética da reação enzimática que ocorre na membrana porosa 2 conforme o mecanismo de Michaelis-Menten em $\mathrm{m}^{3} \cdot \mathrm{mol}^{-1} \cdot \mathrm{s}^{-1}$; $\mathrm{E}_{\mathrm{t}}$ é concentração total de enzima

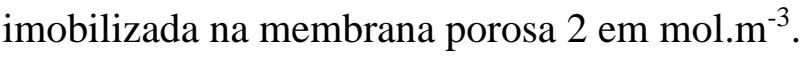

- $\quad$ Balanço para o produto $\mathrm{P}_{1}$ da reação na membrana porosa 2:

$P_{2} \frac{\partial C_{p 1}}{\partial t}-\nabla\left(D_{p m 2} C_{p 1}\right)=R_{s} \quad t>0 \quad \delta_{1}<x<\delta_{2} ; C_{p 1}(x, t=0)=0 \quad t=0 \quad \delta_{1}<x<\delta_{2}$

$\frac{\partial C_{p 1}}{\partial x}=0 \quad t>0 \quad x=\delta_{1} ; \quad \frac{\partial C_{p 1}}{\partial x}=\frac{I(t)}{F \times n \times D_{p m 2}} \quad t>0 \quad x=\delta_{2} ;$

$q_{p 1}(x, t)=\frac{C_{p 1, \max }^{*} K_{L, p 1}}{1+C_{p 1}^{*}(x, t) K_{L, p 1}} \quad t>0 \quad \delta_{1}<x<\delta_{2}$

onde $\mathrm{C}_{\mathrm{p} 1}(\mathrm{x}, \mathrm{t})$ é o perfil de concentração de efluente ao longo da espessura da membrana porosa 2 , em mol.m ${ }^{-3} ; \delta_{2}$ é a posição da membrana porosa $2 \mathrm{em} \mathrm{x}=\delta_{2}$, em $\mathrm{m} ; \mathrm{D}_{\mathrm{sm} 2}$ é o coeficiente de difusão do efluente na membrana porosa $2, \mathrm{em} \mathrm{m}^{2} \cdot \mathrm{s}^{-1} ; \mathrm{D}_{\mathrm{pm} 2}$ é o coeficiente de difusão do produto na membrana porosa $2, \mathrm{em} \mathrm{m}^{2} \cdot \mathrm{s}^{-1} ; \mathrm{I}(\mathrm{t})$ é a densidade de corrente de saída do eletrodo de trabalho A.m ${ }^{-2}$; F é a constante de Faraday em C.mol ${ }^{-1}$, n é o número de elétrons; $\mathrm{q}_{\mathrm{p} 1}(\mathrm{x}, \mathrm{t})$ é a concentração de produto adsorvido por unidade de massa de adsorvente, em $\mathrm{g} / \mathrm{g} ; \mathrm{C}_{\mathrm{p} 1}{ }^{*}(\mathrm{x}, \mathrm{t})$ é a concentração no equilíbrio do produto em solução, em mol.m ${ }^{-3} ; \mathrm{C}_{\mathrm{p} 1 \text {,max }}^{*}$ é o parâmetro que representa a concentração máxima de adsorção na monocamada, em mol.m 3. $K_{L, p l}$ é o parâmetro relacionado com a energia de adsorção e dependente da temperatura, em $1 . \mathrm{g}^{-1}$ 


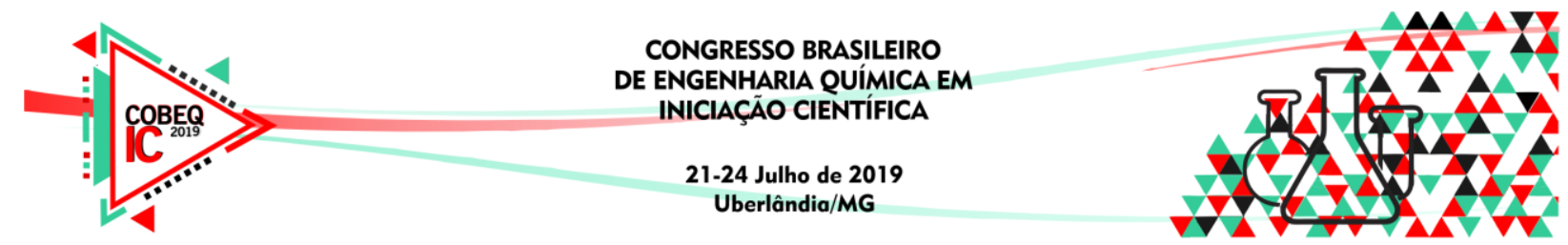

- Balanço de carga no eletrodo de nanopartícula de cobre: A densidade de corrente de saída do eletrodo de trabalho $\mathrm{I}(\mathrm{t})$ depende do fluxo de elétrons da reação de oxirredução que acontece na interface entre a membrana porosa 2 e o eletrodo de trabalho em $\mathrm{x}=\delta_{2}$. Nessa interface, também há interferência da espécie mediadora, produto $\mathrm{P}_{1}$, o qual é eliminado na superfície do eletrodo. Utilizou-se a lei de Faraday e a lei de Fick para expressar a densidade de corrente de saída, I(t), do fluxo de elétrons no eletrodo quando este é oxidado no eletrodo de trabalho:

$$
I(t)=n \times F \times D_{p 1 m 2} \times\left.\frac{\partial P_{1}}{\partial x}\right|_{x=\delta_{2}}
$$

\section{Resultados e Discussão}

Os resultados foram obtidos através das simulações com o COMSOL Multiphisics. A Tabela 1 apresenta os parâmetros que foram utilizados para alimentar o COMSOL e realizar as simulações.

Tabela 1 - Parâmetros utilizados na simulação

\begin{tabular}{|c|c|c|c|c|c|c|c|c|c|c|}
\hline $\begin{array}{c}\delta \\
\mathrm{M}\end{array}$ & $\begin{array}{c}\delta \\
\mathrm{m}\end{array}$ & $\begin{array}{c}\mu \\
\mathrm{s}^{-1}\end{array}$ & $\begin{array}{c}\mathrm{n} \\
\mathrm{s} . \mathrm{u} .\end{array}$ & $\begin{array}{c}\mathrm{S}_{0} \\
\mathrm{~mol} / \mathrm{m}^{3}\end{array}$ & $\varepsilon_{1}$ & $\varepsilon_{2}$ & $\begin{array}{c}\mathrm{D}_{\mathrm{sm} 1} \\
\mathrm{~m}^{2} / \mathrm{s}\end{array}$ & $\begin{array}{c}\mathrm{D}_{\mathrm{sm} 2} \\
\mathrm{~m}^{2} / \mathrm{s}\end{array}$ & $\begin{array}{c}\mathrm{D}_{\mathrm{mP} 2} \\
\mathrm{~m}^{2} / \mathrm{s}\end{array}$ & $\begin{array}{c}\mathrm{K}_{\mathrm{m}} \\
\mathrm{mol} / \mathrm{m}^{3}\end{array}$ \\
\hline $5 \times 10^{-3}$ & $5 \times 10^{-3}$ & $17 \times 10^{-9}$ & 2 & 0.1 & 0.1 & $0.1 \times \varepsilon_{1}$ & $1 \times 10^{-9}$ & $0.1 \times \mathrm{D}_{\mathrm{sm} 1}$ & $0.1 \times \mathrm{D}_{\mathrm{sm} 1}$ & $5 \times 10^{-6}$ \\
& & & & $\begin{array}{c}1.0 \\
10.0\end{array}$ & & & 0.5 & $0.5 \times \varepsilon_{1}$ \\
& & & & & & & & \\
\end{tabular}

A simulação foi dividida em três estudos. A porosidade na primeira e na segunda membrana variou em $\varepsilon_{1}=\varepsilon_{2}=0.1 ; 0.5$ e 0.9 . Todos os ensaios foram feitos no $t=0.9 \mathrm{~s}$. Os valores de $\mathrm{k}_{\mathrm{p} 1}$ e $\mathrm{k}_{\mathrm{p} 2}$ foram $10^{3}$ e $10^{-3}$, respectivamente. A massa específica do biofilme, $\rho$, foi utilizada como sendo $1400 \mathrm{~kg} \cdot \mathrm{m}^{-3}$, e os valores de $\mathrm{K}_{\mathrm{L}}$ foram considerados: $\mathrm{K}_{\mathrm{L}, \mathrm{s} 2}=\mathrm{K}_{\mathrm{L}, \mathrm{s} 1}=$ $0,0001 \mathrm{~m}^{3} \cdot \mathrm{mol}^{-1}$ e $\mathrm{K}_{\mathrm{L}, \mathrm{p} 1}=0,1 \mathrm{~m}^{3} \cdot \mathrm{mol}^{-1}$.

$1^{\circ}$ Estudo: Verificação da influência da porosidade nas membranas sob concentração de efluente inicial $C_{s 1}(x, 0)=0.1 \mathrm{~mol} . \mathrm{m}^{-3}$

Quadro 1 - Gráficos referentes a $\mathrm{S}_{0}: 0.1 \mathrm{~mol}^{-3} ; \varepsilon_{1}: 0.1 ; 0.5 ; 0.9 \varepsilon_{2}: 0.1 ; 0.5 ; 0.9$

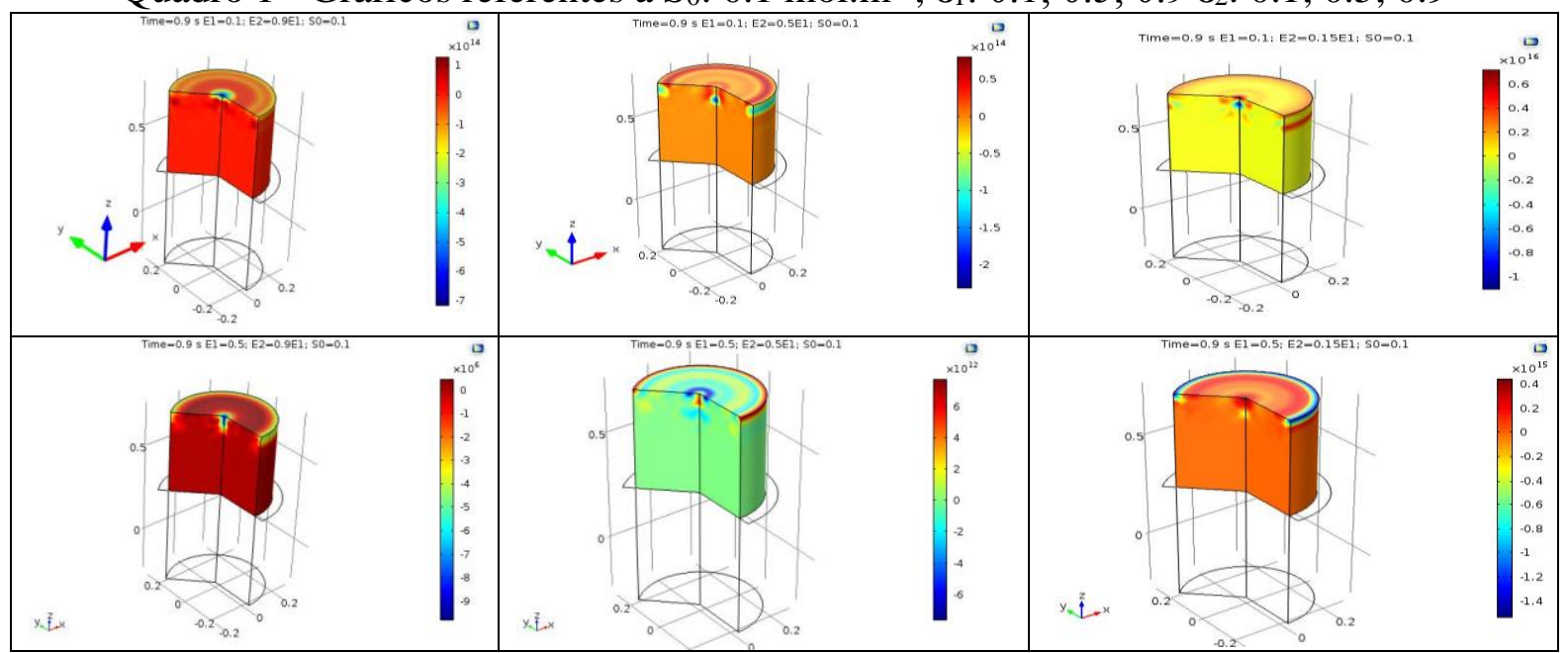




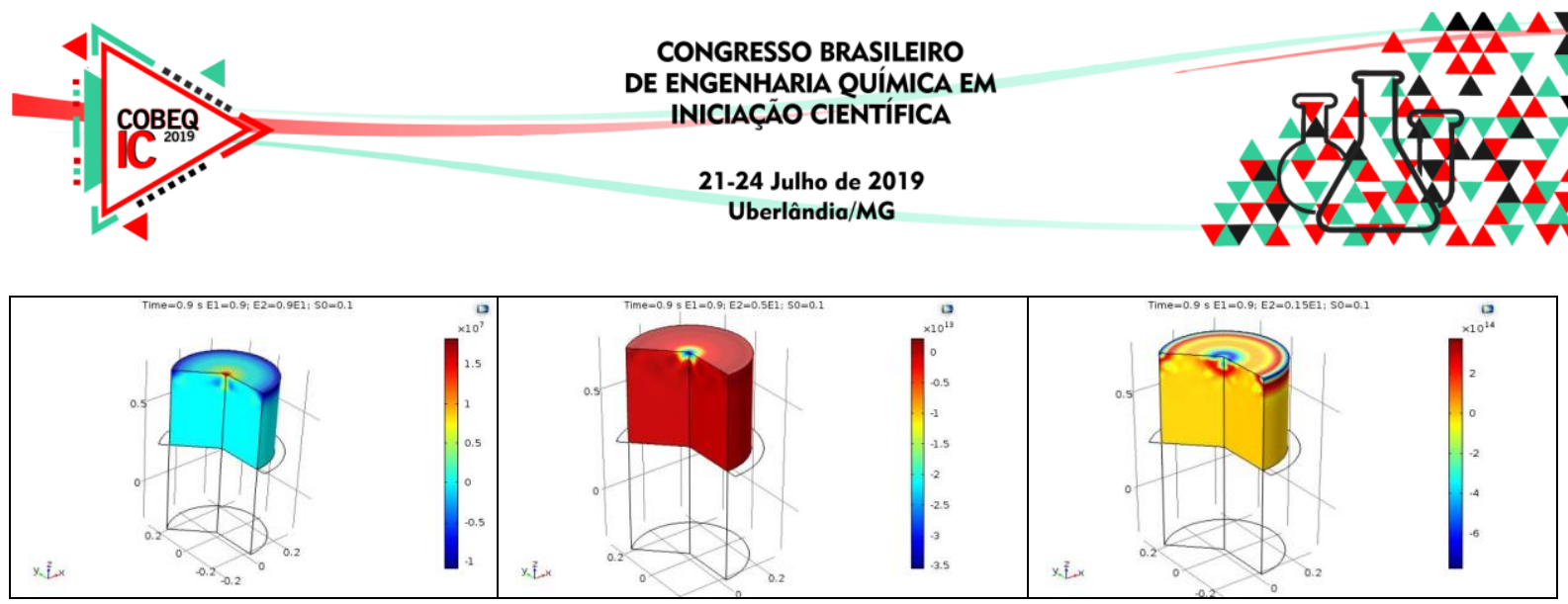

Observa-se no Quadro 1, que para $\varepsilon_{2}=0.9$ e a porosidade $\varepsilon_{1}=0.5$ possui maior eficiência, porém, devido à elevada porosidade na primeira membrana e baixa concentração de substrato, o sinal emitido é pequeno. Para $\varepsilon_{2}=0.5$, a porosidade $\varepsilon_{1}=0.5$ na primeira membrana permite sinal mais eficiente para este estudo.

Para o segundo estudo (Quadro 2), a concentração de substrato em $S_{0}=1.0\left(\mathrm{~mol} / \mathrm{m}^{3}\right)$, assim, quando a porosidade estava em $\varepsilon_{2}=0.9$, o sinal foi ótimo para $\varepsilon_{1}=0.1$, consideramos então que houve adsorção do substrato ao biossenssor devido à porosidade.

Tomando o substrato para $S_{0}=10 \mathrm{~mol} . \mathrm{m}^{-3}$ (Quadro 3), com a porosidade na segunda membrana em $\varepsilon_{2}=0.9$, foi mais eficaz com a porosidade $\varepsilon_{1}=0.9$ na primeira membrana. Quando a porosidade esteve em $\varepsilon_{2}=0.5$, o sinal foi observado em $\varepsilon_{1}=0.1$, porém, sinal pouco eficiente.

$2^{o}$ Estudo: Verificação da influência da porosidade nas membranas sob concentração de efluente inicial $C_{s 1}(x, 0)=1.0$ mol. $\mathrm{m}^{-3}$

Quadro 2 - Gráficos referentes a $\mathrm{S}_{0}: 1.0 \mathrm{~mol}^{-3} ; \varepsilon_{1}: 0.1 ; 0.5 ; 0.9 \varepsilon_{2}: 0.1 ; 0.5 ; 0.9$

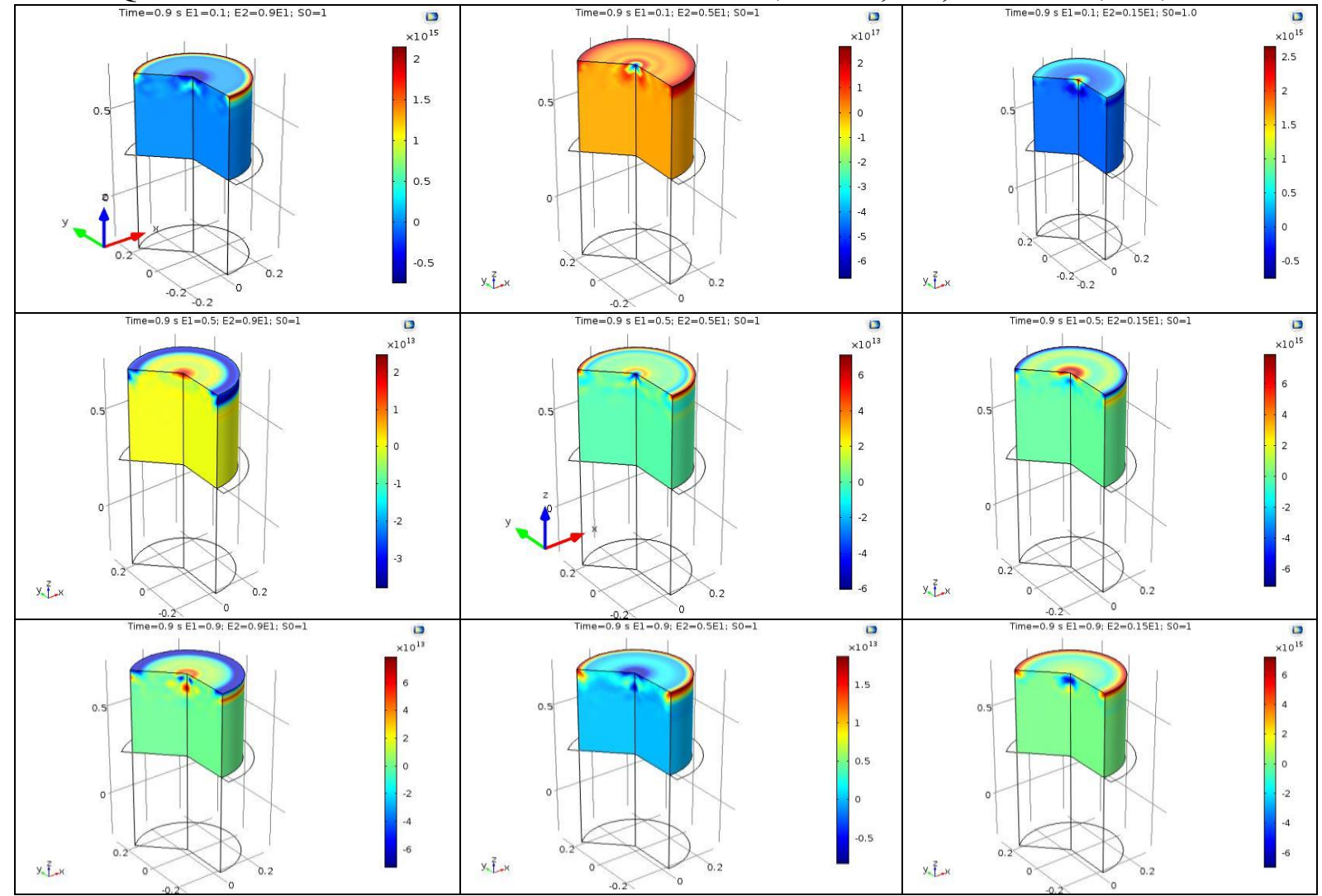




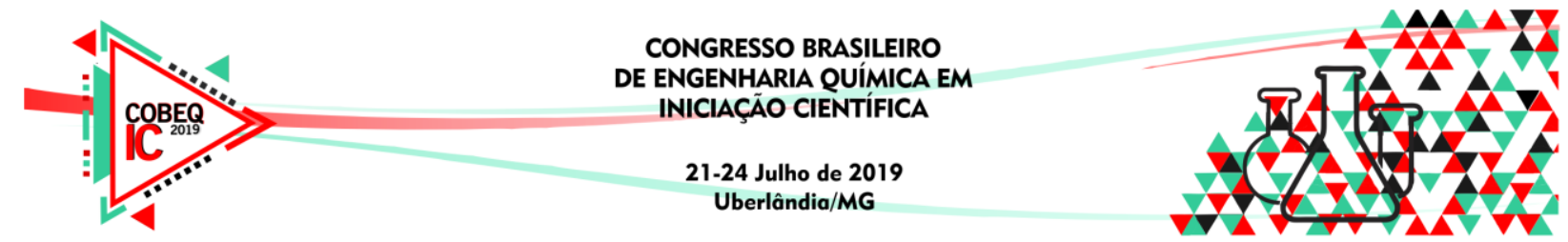

$3^{\circ}$ Estudo: Verificação da influência da porosidade nas membranas sob concentração de efluente inicial $C_{s 1}(x, 0)=10.0$ mol. $\mathrm{m}^{-3}$

Quadro 3 - Gráficos referentes a $S_{0}=10$ mol.m $\mathrm{m}^{-3} ; \varepsilon_{1}=0.1 ; 0.5 ; 0.9$ e $\varepsilon_{2}=0.1 ; 0.5 ; 0.9$

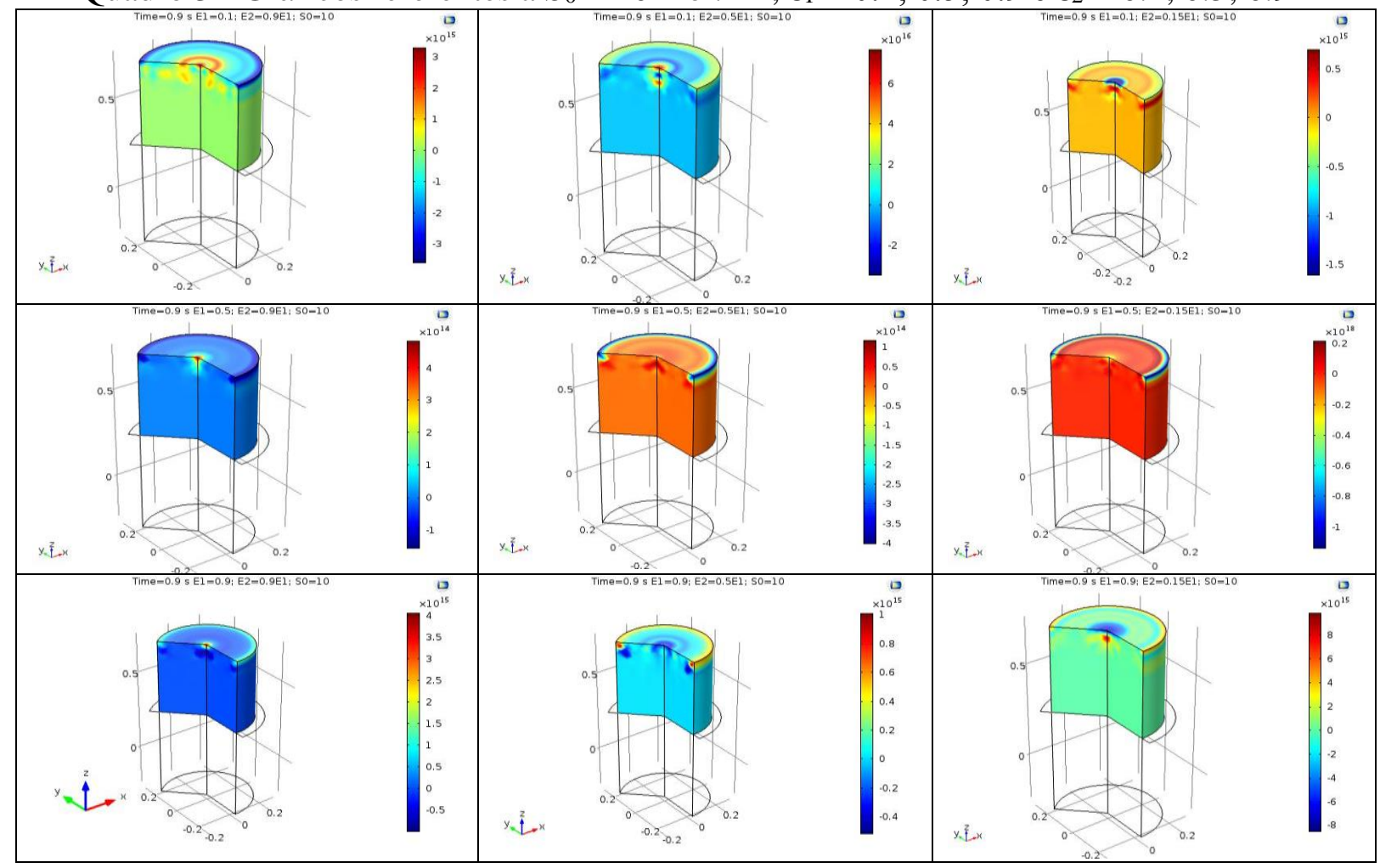

\section{Conclusões}

Do estudo desenvolvido observou-se que a difusão de efluente foi mais eficiente quando a porosidade foi $\varepsilon=0.5$ para ambas as membranas. Pode-se ressaltar que não é bom concentrações muito altas ou baixas de substrato, preferindo assim, $\mathrm{S}_{0}=1.0 \mathrm{~mol} . \mathrm{m}^{-3}$. Esse resultado se confirma pela isoterma de Langmuir, afirmando que a adsorção precisa ocorrer de forma homogênea e que a porosidade precisa ser igual ao longo do estudo.

\section{Referências Bibliográficas}

OLIVEIRA, P. H. R. Métodos de Preparação Industrial de solventes e Reagentes Químicos. Revista Virtual de Química ISSN 1984-6835; Volume 7, número 4. 12 de abril de 2015

ZHANG, Z.; Zhou, J.; Du, X. Electrochemical Biosensors for Detection of Foodborne Pathogens. Micromachines 2019, 10, 222.

WANG Y, Xu H, Zhang J, Li G. Electrochemical Sensors for Clinic Analysis. Sensors. 2008;8(4):2043-2081. 2008 Mar 27. 